\title{
Evaluation of Fluorescent Plasma Markers for in vivo Microscopy of the Microcirculation
}

\author{
Kimberley J. Reeves Zoë L.S. Brookes Malcolm W.R. Reed Nicola J. Brown \\ Microcirculation Research Group, Academic Unit of Surgical Oncology, Department of Oncology, \\ University of Sheffield, Sheffield, UK
}

\section{Key Words}

Fluorochrome $\cdot$ Macromolecular leak $\cdot$ Fluorescent-protein conjugate $\cdot$ In vivo microscopy $\cdot$ Dorsal microcirculatory chamber $\cdot$ Endothelial integrity $\cdot$ Microcirculation

\begin{abstract}
This study evaluated four fluorescent-protein conjugates to monitor microcirculatory variables using the murine cremaster muscle and determined acute and long-term responses to repeated administration of FITC-BSA [conjugated at the University of Sheffield (UoS)] within a dorsal microcirculatory chamber (DMC) in rats. For analysis of the cremaster muscle, male $\mathrm{C} 3 \mathrm{H} / \mathrm{HeN}$ mice were anaesthetized, the cremaster muscle was exteriorized, then TRITC-BSA, TRITC-dextran, FITCBSA, FITC-BSA (UoS) or FITC-dextran $(0.25 \mathrm{ml} / 100 \mathrm{~g})$ were administered systemically. The microcirculation was viewed with epi-illumination every $10 \mathrm{~min}$ for $120 \mathrm{~min}$. For analysis of the DMC, male Wistar rats were implanted with the chamber. Three weeks later, FITC-BSA (UoS) was administered systemically, and the microcirculation response was monitored using three different protocols. In addition, in vitro stability of fluorescent conjugates was measured over $8 \mathrm{~h}$. With re-
\end{abstract}

gard to the cremaster muscle, initially no differences in interstitial fluorescence or vessel diameter were observed between the four fluorescent conjugates. By the end of the study, interstitial fluorescence from TRITC-dextran, FITC-dextran and FITC-BSA (Sigma) was significantly $(p<0.05)$ increased compared to FITC-BSA (UoS). With regard to the DMC, there was no interstitial fluorescence leakage after 180 min or 5 weeks despite repeated administration, but a significant $(p<0.05)$ leak was detected between 4 and 24 h. FITC-BSA (UoS) was the most stable fluorescent conjugate both in vitro and in vivo and was comparable with other conjugates for evaluating skeletal muscle microcirculation using fluorescent in vivo microscopy.

Copyright $\odot 2012$ S. Karger AG, Basel

\section{Introduction}

For many years our studies have employed FITC-albumin (BSA) to investigate interstitial fluorescence in both control and experimental models, including ischaemiareperfuson injury, sepsis and cancer [1-3]. There has been debate in the literature as to the most biologically relevant

\section{KARGER}

Fax +4161306 1234 E-Mail karger@karger.ch www.karger.com (c) 2012 S. Karger AG, Basel

$1018-1172 / 12 / 0492-0132 \$ 38.00 / 0$

Accessible online at:

www.karger.com/jvr
Dr. Kimberley Reeves

Microcirculation Research Group, Academic Unit of Surgical Oncology

Floor K, University of Sheffield School of Medicine and Biomedical Sciences Sheffield, S10 2RX (UK)

Tel. +44 114271 1707, E-Mail k.j.reeves@ sheffield.ac.uk 
fluorescent-macromolecule conjugate to use in microvascular-based research [4-6]. Despite previously published evidence that FITC-albumin induces phototoxicity [7$11]$ and photobleaching $[12,13]$, this has not been apparent in our previous experiments and is the subject of this study.

Fluorescein isothiocyanate (FITC) is probably the most frequently used fluorescent dye for study of the microcirculation, due to its solubility in aqueous media, relatively high quantum yield, availability and low cost $[9$, 13-17]. However, one of the major limitations of FITC conjugates is photobleaching, both in vitro and in vivo, whereby following light activation, the molecule is no longer visible $[12,13]$. Recently, there has been increased use of tetramethylrhodamine isothiocyanate (TRITC) in studies of the microcirculation. Compared to FITC, TRITC demonstrates enhanced stability and is less sensitive to environmental conditions such as $\mathrm{pH}[4,18,19]$. Other fluorescent dyes used less extensively include Texas Red, Oregon Green-514 and dichlorotriazinyl aminofluorescein [18-21]. Two macromolecules are frequently conjugated to the fluorescent dyes to evaluate microvascular barrier function, namely BSA [4] and dextran, the latter being characterized by a range of molecular weights (MWs), good solubility and low toxicity $[8,17]$. Without protein conjugation, fluorescent probes, due to their size and charge, leak through the endothelial barrier into the interstitium.

The use of fluorescent-conjugated macromolecules to study the microcirculation in vivo is based on two essential assumptions, namely that (1) the fluorescent conjugation does not alter the physicochemical properties of the macromolecules and (2) the fluorescent dye does not directly alter the physicochemical properties of the endothelial barrier, i.e. that the conjugates are inert in terms of the microcirculation. However, there is evidence that the light required to activate the fluorescent molecule may result in microvascular dysfunction due to phototoxicity, which appears to involve, at least in part, damage by reactive oxygen species [6,22,23]. Recently, a number of comparative studies have been performed to address these issues by investigating the properties of less well characterized commercially prepared fluorescent conjugates compared to FITC-albumin. Bingaman et al. [4], using FITC, TRITC, Oregon Green, dichlorotriazinyl aminofluorescein and Texas Red in vitro, demonstrated that addition of the macromolecule BSA to FITC resulted in changed physicochemical characteristics, including MW, charge and isoelectric point, which were not altered by controlling the experimental conditions. Thus, modu- lation of the physicochemical properties of the protein may alter protein mobility across the endothelial barrier and hence subsequent localization in the surrounding tissue interstitium. This may potentially result in incorrect conclusions relating to either barrier function or the nature of unlabelled protein interactions.

The second potential problem relating to the use of florescent dye conjugates to study the microcirculation is that of phototoxicity, which has been demonstrated both in vitro and in vivo, in particular for commercially prepared FITC-BSA [7-11]. Few studies have focused on whether the nature of the conjugates affects interpretation of the results [12-23]. In general, phototoxicity is related to the concentration of the fluorescent conjugate, excitation intensity, duration of exposure and the light dose used for activation $[4,9,20]$. Damage is related to the induction of reactive oxygen species, which is reduced by the administration of inhibitors [24]; this may not be immediately evident and is also cumulative [20]. Phototoxicity may be limited by reducing the fluorescent conjugate concentration and the light intensity, in addition to modifying the physicochemical characteristics of the dye [4, 6 , 9]. A comparative in vitro study using commercially available fluorescent dye conjugates demonstrated that during continuous light excitation, phototoxicity occurred during shorter time periods with FITC-BSA when compared to 4,4-difluoro-5,7-dimethyl-4-bora-3a,4a-diaza-indacene-3-propioic acid (BODIPY)-FL-BSA, Texas Red-BSA and TRITC-BSA [20]. These studies emphasize the importance of controlling the light excitation dose and intensity and highlight the need to evaluate dye content and the nature of the fluorescent conjugate when selecting fluorescent molecules for microvascular studies.

This study aimed to evaluate the hypothesis that our 'in-house' preparation of FITC-BSA is a suitable fluorescent conjugate for intravital microscopy when compared with other routinely used fluorescent markers and that deconjugation results in the development of interstitial fluorescence. Initially, the in vitro stability of different fluorescent dye conjugates was evaluated. Then we performed an in vivo comparative evaluation of the stability of microvascular function in the cremaster muscle, as assessed by diameter and vascular fluorescence, using commercial and 'in-house' fluorescent conjugates. Finally, we assessed whether repeated administration of FITC-BSA and varying the illumination duration resulted in microvascular instability using the in vivo dorsal microcirculatory chamber (DMC) skeletal muscle model in conscious animals. 


\section{Materials and Methods}

Animals

Experiments were performed on male $\mathrm{C} 3 \mathrm{H} / \mathrm{HeN}$ mice $(\mathrm{n}=78)$ weighing 20-30 g (model 1: cremaster muscle) and Wistar rats ( $\mathrm{n}=18$ ) weighing $80-280 \mathrm{~g}$ (model 2: DMC) obtained from the University of Sheffield (UoS) Field Laboratories and held in the animal facility for at least 1 week before experimentation. Animals were exposed to a 12/12-hour light/dark cycle in a humidityand temperature-controlled environment and allowed access to water and food ad libitum. All procedures were performed in accordance with the UK Home Office Animal Procedures Act (1986), Project License numbers 40/2343 (model 1) and 40/2110 (model 2).

\section{Protein-Fluorescent Conjugation}

FITC-dextran (MW 70 kDa; FD-70), TRITC-dextran (MW 65-76 kDa; T1162), FITC-BSA (Sigma; MW 66 kDa; A9771), FITC (F7250), TRITC (T3163) and BSA (MW 66 kDa; A7030) were purchased from Sigma-Aldrich (Poole, UK). Dextran and BSA have similar MWs.

\section{FITC-BSA Conjugation (UoS)}

BSA was conjugated to FITC as previously described $[3,25]$. Briefly, $0.037 \mathrm{~g}$ of FITC isomer I and $2 \mathrm{~g}$ of BSA were dissolved in $20 \mathrm{ml}$ of bicarbonate solution $\left(0.12 \mathrm{~g} \mathrm{Na}_{2} \mathrm{CO}_{3}, 0.74 \mathrm{~g} \mathrm{NaHCO}_{3}\right.$, water, $\mathrm{pH}$ 9.0). The covered container of conjugate was mixed and then stirred for $24 \mathrm{~h}$, followed by centrifugation at $6,000 \mathrm{~g}$ for 10 min. The supernatant was placed into washed cellulose dialysis tubing (MW 12,000; D-0530, Sigma-Aldrich), which was then immersed in 2 litres of Nairn's solution (17 $\mathrm{g} \mathrm{NaCl}, 0.692 \mathrm{~g}$ $\mathrm{NaH}_{2} \mathrm{PO}_{4} \cdot \mathrm{H}_{2} \mathrm{O}, 2.14 \mathrm{~g} \mathrm{Na}_{2} \mathrm{HPO}_{4}$, pH 7.4) and stirred for $12 \mathrm{~h}$. The Nairn's solution was changed 24 h later for 4 litres of fresh Nairn's solution and stirred for a further $12 \mathrm{~h}$. The conjugate was stored in the dark as frozen $0.5-\mathrm{ml}$ aliquots until required.

\section{TRITC-BSA Conjugation}

BSA conjugated to TRITC was prepared as previously described [12]. Briefly, $60 \mathrm{mg}$ of BSA was dissolved in $10 \mathrm{ml}$ of $0.05 \mathrm{M}$ borate buffer $\left(0.049 \mathrm{M} \mathrm{Na}_{2} \mathrm{~B}_{4} \mathrm{O}_{7} \cdot \mathrm{H}_{2} \mathrm{O}, \mathrm{pH} 9.24,20^{\circ} \mathrm{C}\right)$ containing $0.4 \mathrm{M} \mathrm{NaCl}$ and placed in dialysis tubing (MW 12,000; D-0530, Sigma-Aldrich). Dye solution was prepared by dissolving $10 \mathrm{mg}$ of TRITC in $50 \mathrm{ml}$ of $0.05 \mathrm{M}$ borate buffer. The covered container of dye with the dialysis bag was stirred for $24 \mathrm{~h}$. The labeled material was then dialysed against 2 litres of glucose-free Ringer's solution. The dialysate was changed $24 \mathrm{~h}$ later for 2 litres of fresh glucose-free Ringer's solution. The conjugate was stored in $0.5-\mathrm{ml}$ aliquots in the dark at $-20^{\circ} \mathrm{C}$ until required.

\section{FITC-Dextran, TRITC-Dextran and FITC-BSA}

The three molecules were purchased as conjugated powder (Sigma, UK). A total of $200 \mathrm{mg}$ of either TRITC-dextran or FITCdextran were dissolved in $4 \mathrm{ml}$ of distilled water, and $2 \mathrm{ml}$ each were dialysed as described above. For FITC-BSA (Sigma), $100 \mathrm{mg}$

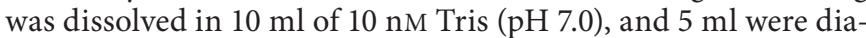
lysed as described above. All samples were stored in $0.5-\mathrm{ml}$ aliquots at $-20^{\circ} \mathrm{C}$ in the dark until required.

The dye content of the fluorescent conjugates conjugated to macromolecules are as follows: FITC-BSA (UoS), 1 molecule of
BSA per 5.9 molecules of FITC; FITC-BSA (Sigma), 1 molecule of BSA per 7-12 molecules of FITC; TRITC-BSA, 1 molecule of BSA per 6 molecules of TRITC; FITC-dextran, 1 molecule of FITC per 1 molecule of glucose, and TRITC-dextran, 1 molecule of TRITC per 100 glucose residues.

\section{Measurement of Bound Fluorescent Conjugate}

The bound fluorescent conjugates of FITC-BSA (Sigma), FITC-BSA (UoS), TRITC-BSA, FITC-dextran and TRITC-dextran were measured in vitro and in vivo. In vitro, the conjugates were measured following incubation at $37^{\circ} \mathrm{C}$ in a water bath for different time periods, i.e. 0, 2, 4 and 8 h. In vivo, FITC-BSA (Sigma), FITC-BSA (UoS), FITC-dextran and TRITC-dextran $(0.25$ $\mathrm{ml} / 100 \mathrm{~g}$ ) were administered into the carotid artery, and blood samples were taken at $0 \mathrm{~h}$ (immediately after injection) and $2 \mathrm{~h}$.

The samples (pre-dialysis and dialysis) from both in vitro and in vivo studies were separated into bound $(>30 \mathrm{kDa})$ and unbound $(<30 \mathrm{kDa})$ fluorescent conjugate fractions using a Centrifree Micropartition device ( $30 \mathrm{kDa}$; Millipore, UK) according to the manufacturer's instructions. Fluorescence was detected using a spectrofluorometer (Perkin Elmer). FITC-BSA and FITC-dextran were excited at $495 \mathrm{~nm}$, and fluorescence was detected between 470 and $650 \mathrm{~nm}$. TRITC-BSA and TRITC-dextran were excited at $547 \mathrm{~nm}$, and fluorescence was detected at $572 \mathrm{~nm}$. Spectrofluorometer readings were compared between bound and unbound fluorescent conjugate fractions.

\section{Surgical Preparation}

Model 1: Cremaster. Mice weighing 20-30 g (age 7 weeks) were anaesthetized with an intra-peritoneal injection of a mixture of Hypnorm (Janssen Pharmaceutical) $0.5 \mathrm{ml} / \mathrm{kg}$, Diazemuls (Dumex) $0.5 \mathrm{ml} / \mathrm{kg}$ and water $[1: 1: 2(\mathrm{v} / \mathrm{v})]$. A tracheotomy was performed, and a Portex cannula beveled at one end was inserted to preserve the airway. An oesophageal thermistor probe (Fluke Ltd., UK) was inserted and connected to a digital thermometer (52II, Fluke Ltd.). The left carotid artery was cannulated and connected to a pressure transducer. The transducer allowed arterial blood pressure to be monitored continuously using WinDaq (DATAQ, USA). The animal was placed on a warming pad (ColeParmer Instruments Co., UK) attached to a Perspex animal board, which allowed maintenance of body temperature $\left(37^{\circ} \mathrm{C}\right)$. The cremaster muscle was prepared as previously described [26].

Model 2: DMC. Rats weighing 80-90 g (age 5 weeks) underwent training which involved placement in a restraining device daily for increasing periods of time up to $10 \mathrm{~min}$ in the 2-week period prior to sterile surgery (weeks 1 and 2). When rats weighed 130-140 g (week 3), they were anaesthetized intra-peritoneally with $0.1 \mathrm{ml} / 100 \mathrm{~g}$ Hypnorm/diazepam (1:1), and the DMC was implanted as previously described $[27,28]$, with modifications by the authors [11]. The animals were then allowed to recover from surgery in week 3 before recommencing training in weeks 4-5. Experiments were then performed in weeks 6-10.

\section{In vivo Microscopy}

Animals were transferred to the stage of an Optiphot-2 Nikon fluorescent microscope (Nikon Ltd., UK) equipped with a tungsten lamp for transmitted light and a mercury arc lamp for epiillumination fluorescent light microscopy. The microscope was horizontally mounted for evaluation in model 2. Transmitted light was used for pre-selecting areas of the microcirculation. Im- 
ages of the preparation were viewed through a $\times 10$ objective $(0.03$ numerical aperture, Nikon). A filter cube interposed into the path of a mercury arc lamp allowed blue (450-490 nm) or green (530$560 \mathrm{~nm}$ ) light to be selected for epi-illumination of the skeletal muscle microcirculation. The excitation power densities ranged from 24.8 to $26.4 \mathrm{~mW}$ for green light and from 16.9 to $19.2 \mathrm{~mW}$ for blue light. Images of the preparation were monitored using a CCD camera (KP-161, Hitachi, UK), displayed on a high-resolution monitor (PVM-1443, Sony, UK) and recorded by a video data recorder (VDR-3000, Holdan Ltd., UK) onto CD-RW (650 MB; Sony) for later off-line analysis. This included the addition of time and date information (DTG1, NG Systems, UK). Animals were allowed an equilibration period of $30 \mathrm{~min}$ before the experimental illumination periods. During this time, the preparation was briefly scanned with low-level transmitted light, and suitable areas of interest (AOIs) were identified for study. In model 1, this involved selecting 3-4 AOIs in the cremaster muscle microcirculation to include all of the following types of vessels in each animal: A1 (100-140 $\mu \mathrm{m}), \mathrm{A} 2(60-100 \mu \mathrm{m}), \mathrm{A} 3(30-60 \mu \mathrm{m})$ and A4 (10-30 $\mu \mathrm{m})$ arterioles, and V1 (110-150 $\mu \mathrm{m}), \mathrm{V} 2(70-110 \mu \mathrm{m}), \mathrm{V} 3(40-70$ $\mu \mathrm{m})$ and V4 (10-40 $\mu \mathrm{m})$ venules.

All vessels included had a straight length of at least $500 \mu \mathrm{m}$. No branches were present in the area of analysis. Interstitial fluorescence was quantified by placing three $90-\mu \mathrm{m}^{2}$ boxes $50 \mu \mathrm{m}$ apart immediately adjacent to the vessel of interest, avoiding areas that included underlying capillaries. The mean fluorescence of the 3 sites was evaluated. Similarly, three $90-\mu \mathrm{m}^{2}$ boxes $50 \mu \mathrm{m}$ apart were placed inside the vessel to quantify intravascular fluorescence. For the subcutaneous maximus muscle microcirculation (model 2), 3 areas were selected using the criteria detailed above to include arterioles and venules (random locations, but in the same location each week) as follows: A1 (70-110 $\mu \mathrm{m})$ and A4 (10-30 $\mu \mathrm{m})$ arterioles, along with V1 (180-250 $\mu \mathrm{m})$ and V4 (10-40 $\mu \mathrm{m})$ venules.

\section{Experimental Groups}

Model 1. Mice were randomly allocated to 5 experimental groups: FITC-BSA (Sigma) $(\mathrm{n}=18)$, FITC-BSA $(\mathrm{UoS})(\mathrm{n}=12)$, FITC-dextran $(\mathrm{n}=18)$, TRITC-dextran $(\mathrm{n}=18)$ and TRITC-BSA $(\mathrm{n}=12)$. For 12 animals in each group $(\mathrm{n}=6$ each for pre-dialysis and dialysis), the fluorescent conjugate $(0.25 \mathrm{ml} / 100 \mathrm{~g})$ was administered into the carotid artery at $\mathrm{t}=0 \mathrm{~min}$. Following a $30-\mathrm{min}$ stabilization period $(\mathrm{t}=-30$ to 0$)$, a baseline recording of the AOI was made at $\mathrm{t}=0$. During illumination with blue light, the AOIs were recorded for $1 \mathrm{~min}$ at 10 -min intervals for a total of $90 \mathrm{~min}$, with a final reading at $t=120 \mathrm{~min}$, when a recording of the entire cremaster preparation was made. As a control, 6 animals in each group were illuminated only at $t=0$ and $t=120$. Mice were also allocated to 2 extra groups, i.e. FITC alone and FITC-BSA. The experimental protocol was as above, except recordings were made every minute for $10 \mathrm{~min}$, as the FITC alone immediately leaked into the interstitium, thus obscuring the preparation.

Model 2. In week 6, conscious rats were randomly allocated to either the 180-min acute study (week 6 only, $n=6$ experiments for $180 \mathrm{~min}$ ), the 24 -hour acute study (week 6 only, $\mathrm{n}=6$ experiments for $24 \mathrm{~h}$ ) or the weekly 180 -min chronic study (weeks $6-10, \mathrm{n}=6$ experiments for the 180-min protocol repeated weekly on 5 occasions). In the 180 -min acute studies, FITC-BSA $(0.25 \mathrm{ml} / 100 \mathrm{~g})$ was administered into the tail vein, and the AOIs were illuminated with blue light for $1 \mathrm{~min}$ every $12 \mathrm{~min}$ for $180 \mathrm{~min}$ (total = $15 \mathrm{~min}$ ). In the 24-hour acute studies, FITC-BSA was adminis- tered into the tail vein, and the microcirculation was illuminated with blue light for $1 \mathrm{~min}$ at $0,1,2,4,8$ and $24 \mathrm{~h}$ (total $=6 \mathrm{~min}$ ). In the chronic studies, the $180-\mathrm{min}$ acute protocol was repeated weekly for 5 weeks.

\section{Measurements}

In anaesthetized mice (model 1), temperature, heart rate and blood pressure were recorded on-line every $10 \mathrm{~min}$ for the entire 120-min experimental period. In model 1, vessel diameters and interstitial and vascular fluorescence were recorded, and in model 2, vessel diameters and interstitial fluorescence were recorded. Measurements were analysed off-line using a Viglen IV/25 computer (Viglen, UK) and an image analysis software package (CapiScope version 3.7.1.0, KK Research Technology Ltd., UK). Each of the 3-4 selected AOIs was intermittently exposed to fluorescent light at specified intervals for a maximum of $1 \mathrm{~min}$ with the aim of limiting any phototoxic damage [3]. For each animal at each time point, measurements of diameter and interstitial fluorescence were made at exactly the same anatomical position. External vessel diameter (including lumen and vessel wall) was measured in microns (micrometres). Fluorescence was quantified using an arbitrary grey scale (0-255), with interstitial fluorescence determined from the mean of 3 distinct areas $\left(900 \mu \mathrm{m}^{2}\right)$ adjacent to the selected vessels and vascular fluorescence determined from the mean of 3 distinct areas $\left(900 \mu \mathrm{m}^{2}\right)$ within the vessel using the image analysis software CapiScope.

\section{Statistical Analysis}

All data are expressed as means \pm standard error of the mean. Measurements of bound fluorescent conjugate are expressed as percentages and evaluated using a paired t test. Statistical significance was evaluated using two-way ANOVA on ranks followed by the Mann-Whitney $U$ test for nonparametric data to determine differences between in vivo experimental groups; within-group variation was assessed using one-way ANOVA on ranks followed by the Wilcoxon test for paired data. All results were considered significantly different at $\mathrm{p}<0.05$.

\section{Results}

\section{Measurement of Bound Fluorescent Conjugate}

Separation studies were performed and a spectrofluorometer was used to assess bound versus unbound fluorescent conjugate in vivo and in vitro. In vitro data demonstrated that FITC-BSA (UoS) had the highest percentage of bound fluorescent conjugate (96\%) at $37^{\circ} \mathrm{C}$, compared to FITC-dextran and TRITC-BSA, with TRITC-dextran (pre-dialysis and post-dialysis) having the lowest percentage of bound fluorescent conjugate (74\%) at all of the time points. However, over the incubation period, all of the fluorescent conjugates became unbound (fig. 1a). In vivo data showed that FITC-BSA (UoS) had the highest percentage of bound fluorescent conjugate both at 0 and $2 \mathrm{~h}$ after injection into the animal, with TRITC-dextran (pre-dialysis and post-dialysis) having 
Fig. 1. a In vitro measurement of the bound fluorescent component of FITC-BSA (UoS), FITC-BSA (Sigma), FITC-dextran, TRITC-BSA and TRITC-dextran (pre-dialysis) at various time points $(0,2,4$ and $8 \mathrm{~h}$ ) after incubation at $37^{\circ} \mathrm{C} .{ }^{*} \mathrm{p}<0.05$ : significant difference compared to FITCBSA ( $t$ test). $n=6$ per group. $\mathbf{b}$ In vivo measurement of the bound fluorescent component of FITC-BSA (UoS), FITC-BSA (Sigma), FITC-dextran and TRITC-dextran (pre-dialysis) at various time points (0 and $2 \mathrm{~h}$ ). ${ }^{*} \mathrm{p}<0.05$ : significant difference compared to FITC-BSA (UoS) (t test). $\mathrm{n}=$ 6 per group.
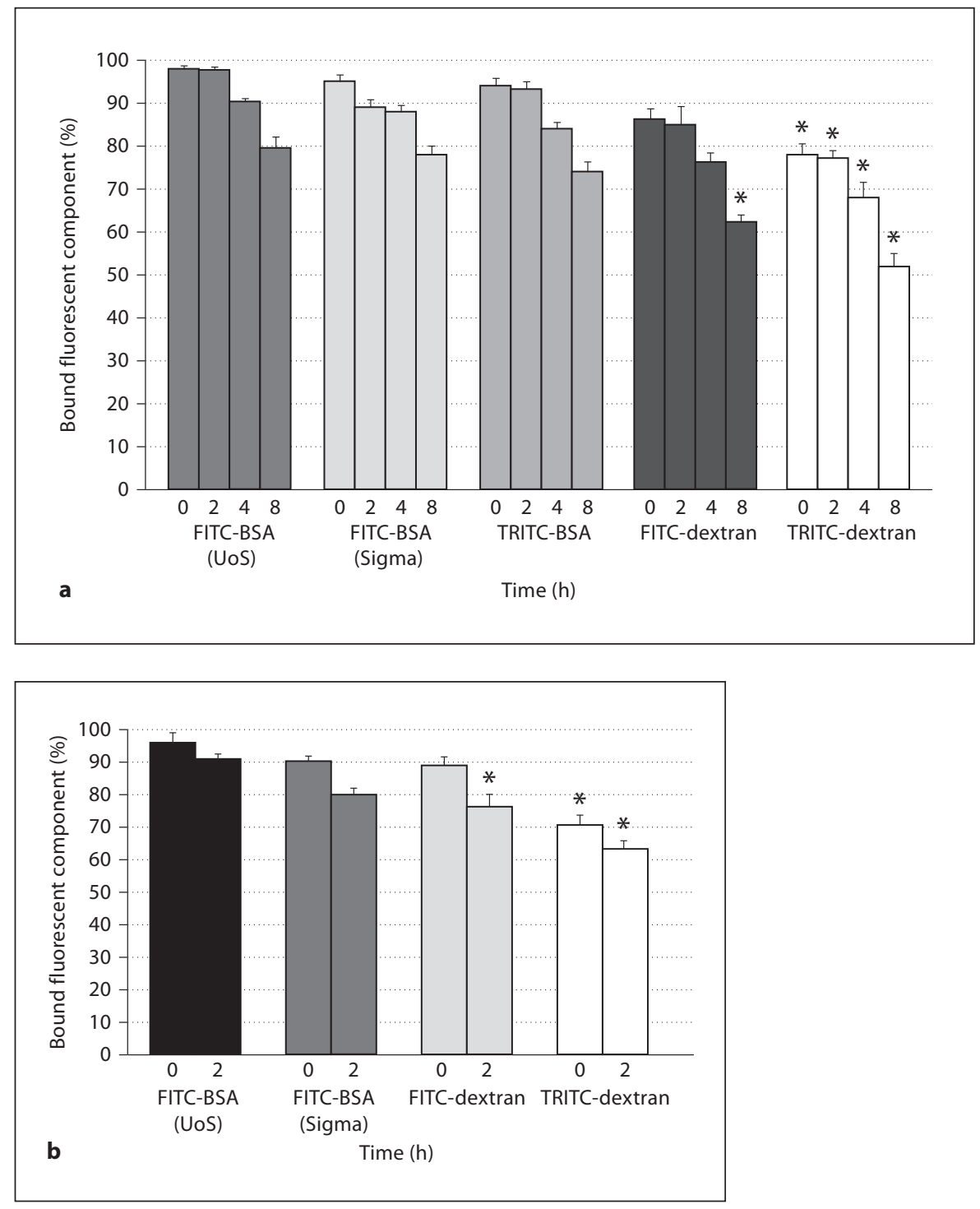

the lowest percentage of bound fluorescent conjugate (fig. 1b). However, over the 2-hour period, all of the fluorescent conjugates became partially deconjugated (fig. 1b), but this was not significant for FITC-BSA (UoS).

\section{Physiological Variables}

There were no differences in oesophageal temperature, cremaster muscle temperature or blood pressure between the control and fluorescent conjugate- and lighttreated groups in either in vivo model.

\section{Model 1: Cremaster - Diameter}

There were no differences in arteriolar diameter or venule diameter between the controls and fluorescent conju- gate- and light-treated groups for any of the fluorescent conjugates for the entire duration of the study (table 1).

\section{Model 1: Cremaster - Interstitial Fluorescence}

Interstitial fluorescence data only from $\mathrm{V} 1, \mathrm{~V} 4, \mathrm{~A} 1$ and $\mathrm{A} 4$ vessels are presented, as the $\mathrm{V} 2, \mathrm{~V} 3, \mathrm{~A} 2$ and $\mathrm{A} 3$ vessels demonstrated the same pattern of response as the firstand fourth-order venules and arterioles, respectively.

TRITC-BSA. At the beginning of the experiment, fluorescent conjugate contained within the vessels was clearly visible, but by $30 \mathrm{~min}$, fluorescence was no longer visible, such that the preparation appeared black. A fluorescent conjugate-conjugate was prepared using the FITC-BSA method, which yielded similar results. Thus 
Table 1. Arteriolar (A) and venule (V) vessel diameters at different time points after administration of FITC-BSA (UoS), FITC-BSA (Sigma), FITC-dextran and TRITC-dextran

\begin{tabular}{|c|c|c|c|c|c|c|c|c|}
\hline & \multicolumn{8}{|c|}{ Vessel diameter, $\mu \mathrm{m}$} \\
\hline & \multicolumn{2}{|l|}{ A1 vessels } & \multicolumn{2}{|l|}{ A4 vessels } & \multicolumn{2}{|l|}{ V1 vessels } & \multicolumn{2}{|l|}{ V4 vessels } \\
\hline & $0 \mathrm{~min}$ & $120 \mathrm{~min}$ & $0 \mathrm{~min}$ & $120 \mathrm{~min}$ & $0 \mathrm{~min}$ & $120 \mathrm{~min}$ & $0 \mathrm{~min}$ & $120 \mathrm{~min}$ \\
\hline FITC-BSA (UoS) & $103.8 \pm 5.4$ & $104.5 \pm 4.1$ & $18.9 \pm 3.6$ & $20.5 \pm 2.8$ & $124.7 \pm 9.7$ & $123.2 \pm 5.1$ & $19.3 \pm 3.4$ & $20.7 \pm 2.5$ \\
\hline FITC-BSA (Sigma) & $100.8 \pm 3.7$ & $101.7 \pm 2.3$ & $18.7 \pm 2.4$ & $19.7 \pm 2.1$ & $125.8 \pm 5.9$ & $124.3 \pm 4.8$ & $15.1 \pm 1.5$ & $15.8 \pm 2.7$ \\
\hline FITC-dextran & $112.3 \pm 4.4$ & $111.2 \pm 6.1$ & $20.2 \pm 2.7$ & $18.9 \pm 1.7$ & $129.7 \pm 9.5$ & $127.8 \pm 10.0$ & $18.6 \pm 2.2$ & $20.0 \pm 2.1$ \\
\hline TRITC-dextran & $106.7 \pm 5.9$ & $103.3 \pm 5.9$ & $19.8 \pm 1.4$ & $20.1 \pm 1.3$ & $123.9 \pm 8.4$ & $125.9 \pm 7.8$ & $19.1 \pm 1.3$ & $20.9 \pm 1.5$ \\
\hline Control & $104.5 \pm 4.7$ & $107.1 \pm 5.3$ & $16.4 \pm 2.4$ & $18.4 \pm 3.0$ & $120.4 \pm 6.1$ & $122.8 \pm 6.3$ & $17.6 \pm 2.0$ & $18.2 \pm 2.4$ \\
\hline
\end{tabular}

data are presented for the three other fluorescent conjugates.

FITC-BSA (UoS). There was no increase in interstitial fluorescence over the 120-min experiment for any of the vessels compared to controls and baseline (fig. $2 \mathrm{a}, \mathrm{b}$ ).

FITC-BSA (Sigma). There was an increase $(\mathrm{p}<0.05)$ in interstitial fluorescence by $60 \mathrm{~min}$ for V1 $(5.4 \pm 1.8)$, by $50 \mathrm{~min}$ for $\mathrm{V} 4(4.2 \pm 2.1)$, by $90 \mathrm{~min}$ for $\mathrm{A} 1(8.6 \pm 9.3)$ and by $80 \mathrm{~min}$ for $\mathrm{A} 4$ vessels $(8.1 \pm 3.8)$ compared to the baseline, which was then maintained for the duration of the study. At $120 \mathrm{~min}$, the FITC-BSA (Sigma) group demonstrated significantly $(\mathrm{p}<0.05)$ more leakage than the FITC-BSA (UoS) group for all vessel types (fig. 2a).

FITC-Dextran. There was no increase in interstitial fluorescence for the initial $40 \mathrm{~min}$, but thereafter there was a significant increase $(\mathrm{p}<0.05)$ adjacent to V1, A1 and A4 vessels compared to baseline $(\mathrm{t}=0)$. This increase occurred at $30 \mathrm{~min}$ in V4 vessels. By $120 \mathrm{~min}$, there was significantly more leakage in the FITC-dextran group compared with the FITC-BSA (UoS) group for all vessel types (fig. 2a, b).

TRITC-Dextran. There was an increase $(\mathrm{p}<0.05)$ in interstitial fluorescence by $30 \mathrm{~min}$ compared to the baseline for all vessel types in all groups, which was maintained for the duration of the study. At $120 \mathrm{~min}$, the TRITC-dextran group demonstrated more leakage in all vessels $(\mathrm{p}<0.05)$ than the FITC-BSA (UoS) group (fig. 2a).

Dialysed Conjugates. FITC-BSA (Sigma), FITC-dextran and TRITC-dextran were all dialysed prior to injection. The dialysed conjugates did not demonstrate reduced interstitial fluorescence (data not presented).

FITC Alone Compared to FITC-BSA (UoS). There was a significant difference in fluorescence between FITC alone and FITC-BSA (UoS) over the $10 \mathrm{~min}$ in all vessel types, which occurred 1 min after administration and peaked after $8 \mathrm{~min}(\mathrm{~V} 118.8 \pm 1.9, \mathrm{~V} 420.4 \pm 2.0$, A1 14.8 \pm 1.4 and A4 $14.9 \pm 0.9$ ). Since FITC is not conjugated to a protein molecule, this leaks immediately from the vessels, hence the shorter duration of this experiment.

\section{Model 1: Cremaster - Vascular Fluorescence}

FITC-BSA (UoS), FITC-BSA (Sigma) and FITC-BSA (Sigma Dialysis). There were no differences in vascular fluorescence over the $120 \mathrm{~min}$ in any of the vessel types compared to control or $t=0$ (fig. $2 c$ ) or between the fluorescent conjugates.

FITC-Dextran. Vascular fluorescence remained constant until $40 \mathrm{~min}$, after which there was an increase in V4 and A4 vessels $(p<0.05)$, which occurred later in V1 and $\mathrm{A} 1$ vessels, at $120 \mathrm{~min}$ (fig. $2 \mathrm{c}$ ).

TRITC-Dextran. There was a significant increase $(\mathrm{p}<$ 0.05 ) in vascular fluorescence by $30 \mathrm{~min}$ compared to baseline for V4 and A4 vessels, but this increase occurred later in V1 and A1 vessels, at $80 \mathrm{~min}$ (fig. 2c).

Dialysed Conjugates. Vascular fluorescence for the dialysed groups showed a similar pattern of results when compared to the undialysed groups.

\section{Model 2: DMC - Diameter}

There were no changes in arteriole diameter (A1 86.5 $\pm 6.3 \mu \mathrm{m}, \mathrm{A} 333.1 \pm 2.9 \mu \mathrm{m}$ ) or venule diameter (V1 $217.7 \pm 14.6 \mu \mathrm{m}, \mathrm{V} 459.2 \pm 6.2 \mu \mathrm{m}$ ) between the control and fluorescent conjugate- and light-treated groups, either for an individual experiment or at the end of the 10week study period.

\section{Model 2: DMC - Interstitial Fluorescence}

In the 180-min acute study, there were no increases in interstitial fluorescence adjacent to the V4 vessels over 180 min when compared to $t=0$ following intermittent epi- 


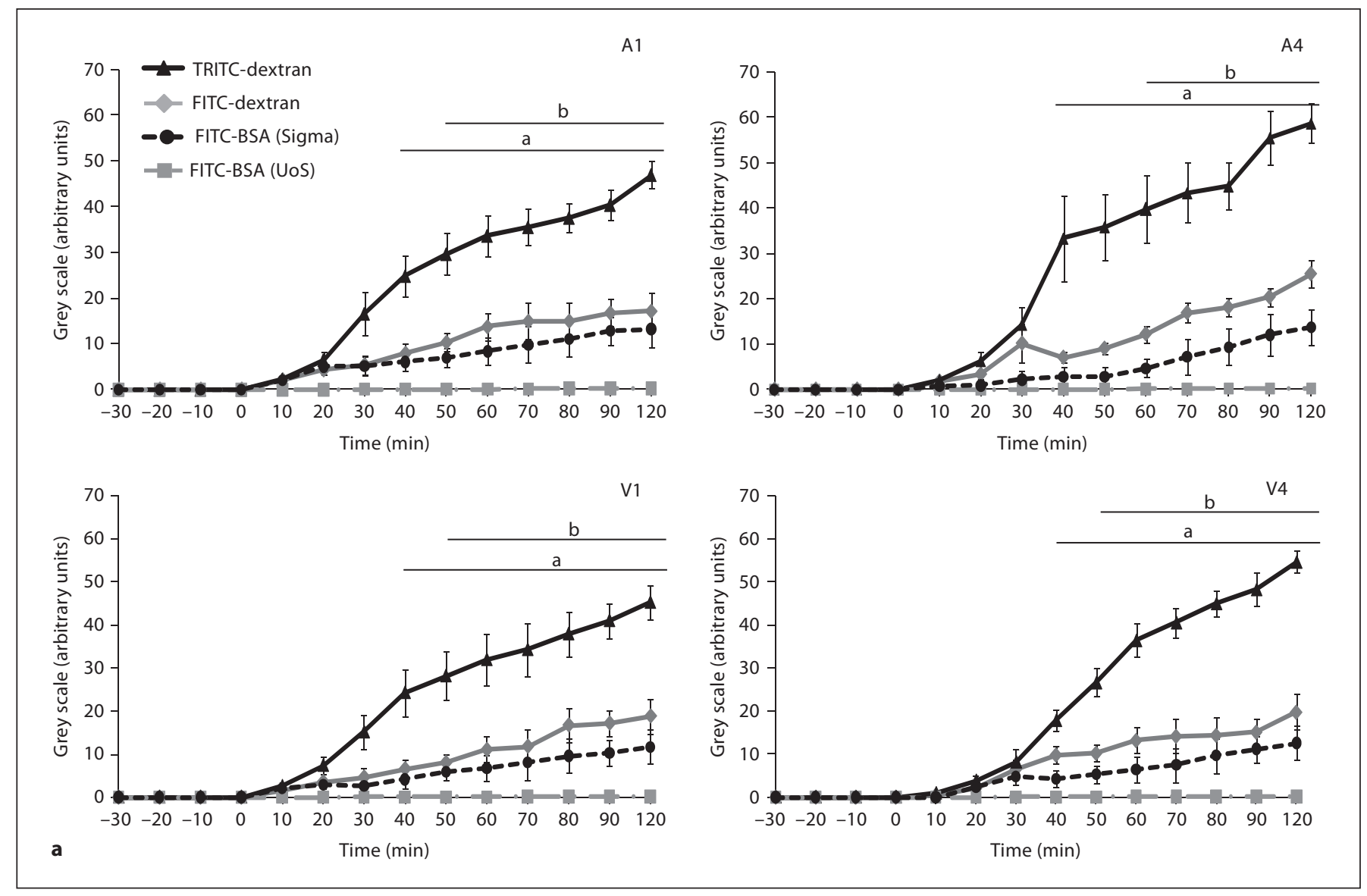

Fig. 2. a The level of fluorescence in the interstitium adjacent to $\mathrm{V} 1, \mathrm{~V} 4, \mathrm{~A} 1$ and $\mathrm{A} 4$ vessels after administration of FITC-BSA (UoS), FITC-BSA (Sigma), TRITC-dextran and FITC-dextran (data nor-

malized to baseline; $\mathrm{n}=6$ /group). ${ }^{\mathrm{a}} \mathrm{p}<0.05$ : significant difference for TRITC-dextran from $t=0$ (Wilcoxon test); ${ }^{b} p<0.05$ : significant difference for FITC-dextran from $t=0$ (Wilcoxon test).
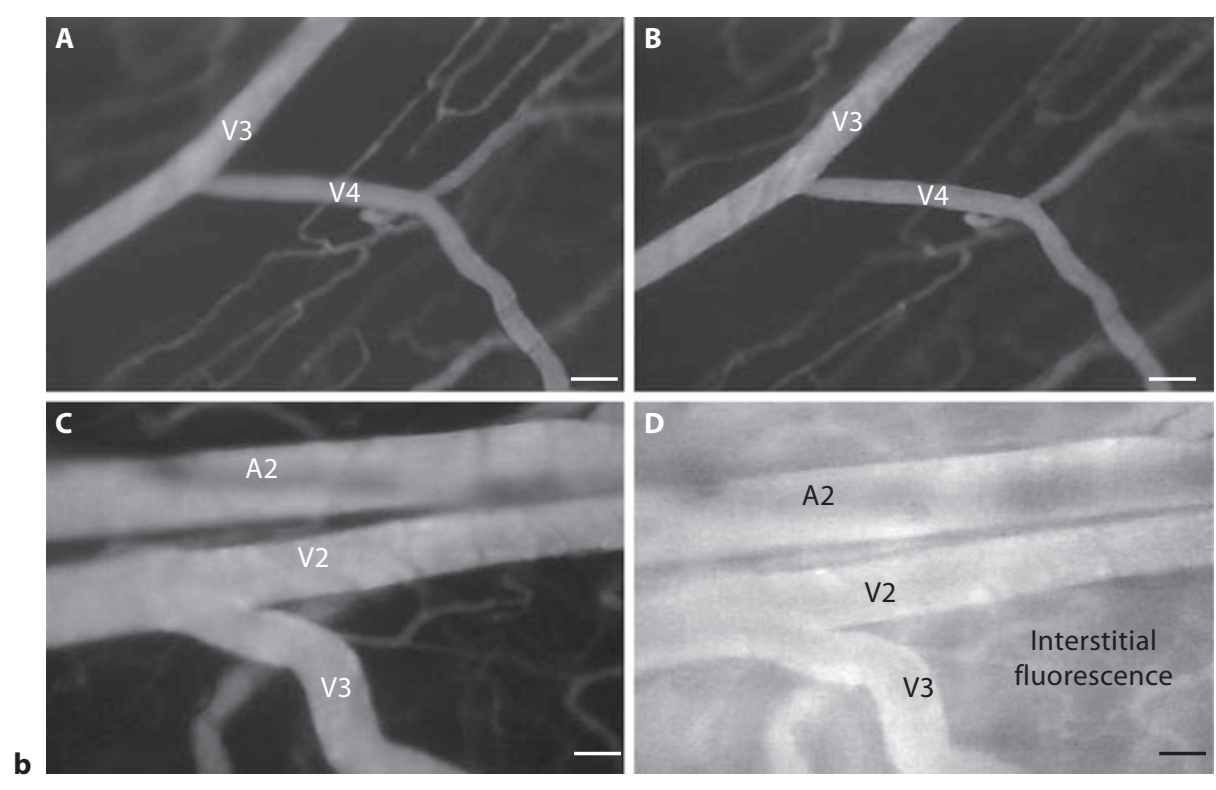

Fig. 2. b A, B V4 vessel at $\mathrm{t}=0 \mathrm{~min}(\mathbf{A})$ and $\mathrm{t}=120 \mathrm{~min}(\mathbf{B})$ after administration of FITC-BSA (UoS) showing no macromolecular leak. Vessels appear white and the interstitium black. C, D V2 and V3 vessels at $\mathrm{t}=0$ (C) after administration of TRITCdextran showing no interstitial fluorescence and at $\mathrm{t}=120$ (D) showing interstitial fluorescence. Scale bar $=50 \mu \mathrm{m}$. 

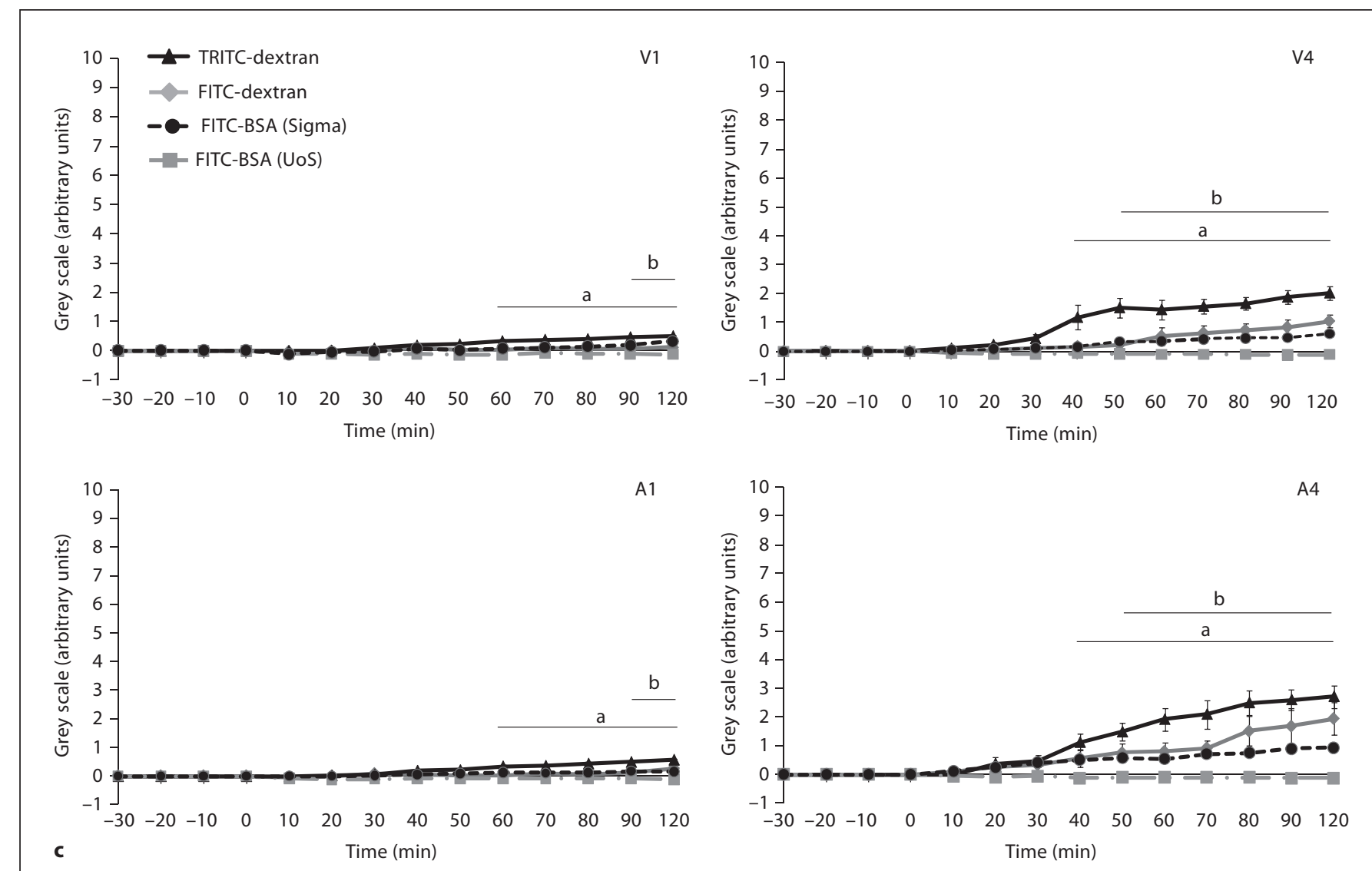

Fig. 2. c Measurement of vascular fluorescence after administration of FITC-BSA (UoS), FITC-BSA (Sigma), TRITC-dextran and FITC-dextran in V1, V4, A1 and A4 vessels (data normalized to

baseline; $\mathrm{n}=6$ ). ${ }^{\mathrm{a}} \mathrm{p}<0.05$ : significant difference for TRITC-dextran from $\mathrm{t}=0$ (Wilcoxon test); ${ }^{\mathrm{b}} \mathrm{p}<0.05$ : significant difference for FITC-dextran from $t=0$ (Wilcoxon test).

illumination for a total of $15 \mathrm{~min}$ ( $\mathrm{p}>0.05$; fig. 3a). However, in the 24-hour acute study, there was significant leakage from the venules at $240 \mathrm{~min}(\mathrm{p}<0.05)$ when compared to $t=0$, despite the lower total exposure time of $4 \mathrm{~min}$. This increased steadily up to $24 \mathrm{~h}$, at which time there had been a total of 6 min of light exposure ( $<<0.05$; fig. $3 b)$. In the repeat studies, there was no increase in leakage on each occasion when compared to $t=0$. This remained the case even at week 10, when the microcirculation had been exposed to 5 weekly doses of FITC-BSA (UoS), with 15 min of intermittent epi-illumination on each occasion ( $p>0.05$; fig. $3 c$ ). Additionally, there appeared to be no side effects or morbidity associated with repeated administration of FITC-BSA (UoS), evaluated according to Home Office regulations (PPL 40/2110), which include vocalization, struggling, licking/guarding, weight loss, piloerection, hunched position and hypothermia.

\section{Discussion}

This is the first in vivo study to investigate the stability of different fluorescent-protein conjugates in vitro and compare microcirculatory responses using two in vivo microvascular models. The investigation was undertaken as previously published data had identified limitations associated with the in vivo use of FITC-BSA, including photobleaching and phototoxicity, and had suggested that alternative protein-fluorescent conjugates may be more appropriate for in vivo evaluation of microvascular responses [4, 9, 18-21]. Despite this evidence, our laboratory had not encountered problems using FITC-BSA in vivo, presumably due to the preparation protocol.

Initially, in vitro studies were performed to evaluate the percentage of bound versus unbound conjugate following incubation at $37^{\circ} \mathrm{C}$ for varying periods up to $8 \mathrm{~h}$. 


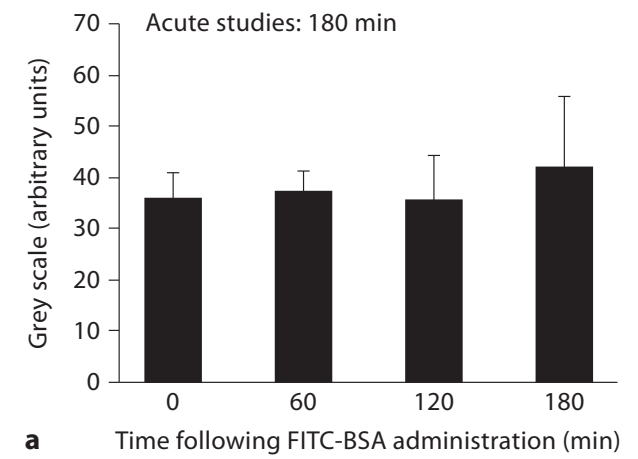

Fig. 3. a Mean $\pm \operatorname{SEM}(n=6)$ interstitial fluorescence adjacent to postcapillary venules (V4) within the skeletal muscle of DMCimplanted rats following FITC-BSA administration and epi-illumination with blue light for $1 \mathrm{~min}$ every $12 \mathrm{~min}$, for a total of 180 min. Studies were performed at week 6 after implantation of the DMC. $\mathbf{b}$ Mean \pm SEM $(n=6)$ interstitial fluorescence adjacent to postcapillary venules (V4) within the skeletal muscle of DMCimplanted rats following FITC-BSA administration and epi-illumination with blue light for $1 \mathrm{~min}$ at $0,1,2,4,8$ and $24 \mathrm{~h}$. Studies were performed at week $6 .{ }^{*} \mathrm{p}<0.05$ : significant difference compared to $\mathrm{t}=0$ (Wilcoxon test). c Mean $\pm \operatorname{SEM}(\mathrm{n}=6)$ interstitial fluorescence adjacent to postcapillary venules (V4) within the skeletal muscle of DMC-implanted rats following FITC-BSA administration and 6 min of epi-illumination with blue light. Studies were performed during weeks $6-9$, with the same light protocol each week over 4 weeks.

Following dialysis of all preparations, FITC-BSA (UoS) demonstrated the highest level of conjugation at all time points investigated. In vivo cremaster muscle microcirculation studies were performed using all the fluorescentprotein conjugates; vascular fluorescence, interstitial fluorescence, vessel diameter and serum levels of bound versus unbound fluorescent conjugate were evaluated. There were no changes in vascular fluorescence with either of the FITC-BSA conjugates, whereas there was a small but significant increase in vascular fluorescence with FITCdextran and TRITC-dextran in fourth-order vessels. The significance of this finding is unclear, although it may in part explain the increase in interstitial fluorescence observed. One possible explanation could be that increased water permeability across the small fourth-order vessels produced a filtration effect, thus increasing vascular fluorescence. Throughout the study, FITC-BSA (UoS) re-
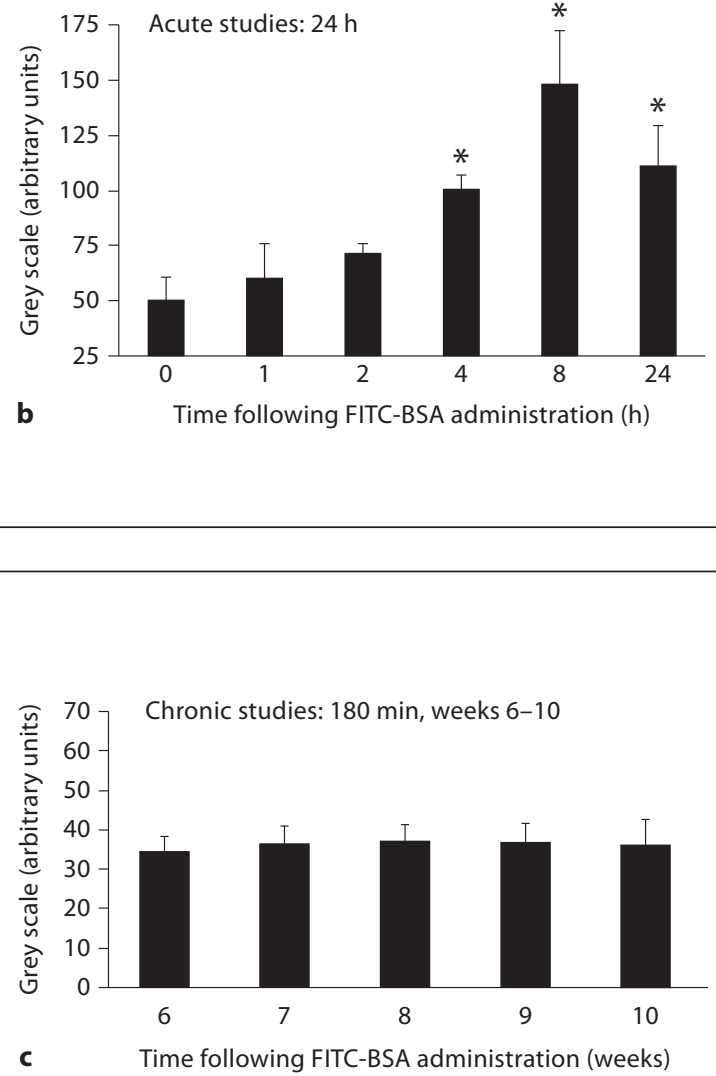

sulted in the least interstitial fluorescence following intermittent excitation using blue light and a total light exposure of $11 \mathrm{~min}$, with no change in vessel diameter for the 2-hour experimental period, suggesting minimal deconjugation and/or phototoxicity. Intermittent excitation of TRITC-dextran resulted in the greatest increase in interstitial fluorescence when compared to the other fluorescent-protein conjugates. Interstitial fluorescence was compared following administration of FITC alone or FITC-BSA; FITC demonstrated immediate leakage (after $1 \mathrm{~min}$ ), whereas FITC-BSA (UoS) remained within the vessels. After $10 \mathrm{~min}$, FITC-BSA remained within the vessels, but FITC alone had leaked, with vessels appearing black with a white edge; in addition, FITC had been removed from the interstitium, presumably by lymph vessels. Serum analysis of the bound versus unbound protein conjugates revealed that despite dialysis, imme- 
diately (0-5 $\mathrm{min}$ ) following in vivo administration, unbound fluorescent molecules were detected, which probably accounts for the increased interstitial fluorescence observed in vivo at the beginning of the study for the FITC-dextran and TRITC-dextran conjugates. This is in addition to the likely deconjugation over time, as demonstrated in the in vitro studies. When considering all fluorescent-protein conjugates, FITC-BSA (UoS) demonstrated the highest percentage of binding in both in vitro and in vivo studies.

A further observation was that systemic administration of TRITC-BSA resulted in intravascular fluorescence for the first $30 \mathrm{~min}$ of observation, following which fluorescence was no longer detectable either in the vessels or the surrounding tissue. This may have been due to extensive initial photobleaching or because following leakage and subsequent clearance there was minimal fluorescence for detection. However, in contrast, TRITC-dextran fluorescence could not be detected. Despite the differences in microvascular variables identified between the BSA groups in vivo, this is unlikely to be due to the ratio of dye to protein, which was similar for each protein-conjugate. However, for the dextran studies, different numbers of glucose molecules (FITC-dextran 1:1, TRITC-dextran 1:100) may in part explain the differences observed between these groups. Therefore, our data suggest that FITC-BSA (UoS) is the most stable fluorescent conjugate for studying interstitial fluorescence and vessel diameter in murine cremaster muscle microcirculation using in vivo microscopy when compared to commercial FITC-BSA, TRITC-dextran and FITC-dextran using intermittent light excitation for a total of $11 \mathrm{~min}$. However, the all fluorescent conjugates benefited from dialysis prior to in vivo administration, which removes a proportion of the unbound component present.

FITC-BSA (UoS) was then evaluated using a second in vivo model, the dorsal window chamber implanted in rats. There was no FITC-BSA (UoS) leakage during the first $180 \mathrm{~min}$ of observation (total of $15 \mathrm{~min}$ of illumination), confirming the findings obtained with model 1 that FITC-BSA (UoS) is a suitable fluorescent conjugate for in vivo microvascular studies, using this particular administration route, conjugation protocol and light dose for up to $3 \mathrm{~h}$. However, in the acute 24-hour studies, although there was no significant interstitial fluorescence between 0 and $2 \mathrm{~h}$, in contrast, between 4 and $24 \mathrm{~h}$, increased leakage was apparent even with reduced total epi-illumination (6 min). These data suggest that FITCBSA is stable for $4 \mathrm{~h}$, after which time deconjugation occurs and results in leakage into the interstitium. Repeat- ed administration studies were also carried out between weeks 6 and 10, during which time the 180-min acute study was performed on 5 occasions, and confirmed that this protocol can be used repeatedly at weekly intervals without causing damage to the microcirculation. Furthermore, no observable long-term side effects occurred in conscious animals in response to repeated FITC-BSA administration.

There are two main potential mechanisms explaining an increase in interstitial fluorescence, namely phototoxicity and deconjugation. Phototoxicity may occur following extensive exposure of a fluorescent conjugate to light. The excitation induces a photochemical response [6], generating reactive oxygen species [22], which leads to the induction of structural changes and damage to the vessel wall resulting in interstitial fluorescence [19]. Microvascular dysfunction and leakage induced by fluorescent conjugate light activation have been reported in hamster cheek pouch with FITC-dextran-70 [8] and in rat cremaster with FITC-BSA [6]. These data are in contrast to those generated in the current study. The likely explanation is that the response is dependent upon the excitation time, with leakage evident following continuous exposure (excitation light) for $10 \mathrm{~min}$ [6], whereas intermittent light exposure (for $1 \mathrm{~min}$ at 10-min intervals) is less damaging to the tissue, with reduced phototoxicity. In addition, we observed no leukocyte or thrombocyte adhesion (in either model), which often occurs following vascular damage $[1,11]$.

An alternative mechanism is that the protein may become unbound and remain in the vasculature, whereas the fluorescent conjugate leaks into the interstitium. Deconjugation, as opposed to phototoxicity, is the most likely explanation for the leakage observed in the current study, as vessel diameters remained unchanged throughout each experiment, and increased venular permeability has previously been shown to be accompanied by increased capillary diameter [10]. However, the sensitivity of the in vivo microscopy system used in the current study may not be sufficient to monitor small but significant changes in capillary diameter. In addition, interstitial fluorescence occurred throughout the cremaster preparation and not just in the activated areas, suggestive of deconjugation. Our in vitro and in vivo binding studies demonstrated a greater proportion of bound fluorescent conjugate for the FITC-BSA (UoS) preparation compared to the other fluorescent conjugate preparations evaluated.

An interesting in vitro study using rat erythrocytes demonstrated that phototoxicity occurs after a shorter 
period of time using continuous excitation of FITC-BSA (Sigma) compared to BODIPY-FL-BSA, Texas Red-BSA and TRITC-BSA. TRITC-BSA also appeared to be the least phototoxic of the four fluorescent dyes tested [20]. Conversely, our results demonstrated no evidence of phototoxicity using FITC-BSA, whereas after $30 \mathrm{~min}$ of intermittent illumination, TRITC-BSA was no longer visible. The differences observed between this and previous studies are probably due to the shorter period of illumination used in the current study, with only brief intermittent exposure to light for a maximum of $1 \mathrm{~min}$ at any one time. Microvascular dysfunction following FITC-BSA administration has previously been shown to be dependent on excitation light intensity [6], with continuous excitation of FITC-BSA for 10 min resulting in leakage, whereas intermittent light exposure $(15 \mathrm{~s}$ at 0,5 and $10 \mathrm{~min}$ ) appeared not to induce damage, with no significant leakage by $60 \mathrm{~min}$ [6]. Another study which investigated the fluorescent-protein conjugate leakage response to L-NAME demonstrated that in control experiments (animals received fluorescent conjugate but no L-NAME and were exposed to intermittent light of 5-15 s), FITC-BSA and TRITC-BSA did not leak over time [20]. The rat DMC acute 180-min study (model 2) confirmed these observations. However, in acute 24hour studies, there was no significant interstitial fluorescence between 0 and $2 \mathrm{~h}$, whereas between 4 and $24 \mathrm{~h}$, increased leakage was apparent even with reduced total epi-illumination, supporting our hypothesis that FITCBSA becomes unbound and leaks from the vasculature after $4 \mathrm{~h}$, rather than damage being induced by photoactivation.

Therefore, in summary on the basis of the current studies, FITC-BSA prepared according to our specified protocol is a useful conjugate for determining interstitial fluorescence in skeletal muscle of different species, as well as in microcirculation preparations in conscious and anaesthetized animals. Both models support previous in vivo studies demonstrating no alterations in interstitial fluorescence of FITC-BSA for up to $4 \mathrm{~h}$ when using intermittent excitation $[1,2,23,29]$. In conclusion, the interstitial fluorescence observed with protein fluorescent conjugates is due to their ability to deconjugate over time, as demonstrated both in vivo and in vitro, which emphasizes the need for careful selection of fluorescent markers depending on the in vivo preparation and protocol under study.

\section{Acknowledgements}

We gratefully acknowledge the support of Sheffield Hospitals Charitable Trust (Grant number: RB104471) and Mrs. Sue Higham for technical assistance with the in vitro fluorescent conjugate experiments.

\section{References}

1 Brookes ZL, Reilly CS, Brown NJ: Response of the rat cremaster microcirculation to hemorrhage in vivo: differential effects of intravenous anesthetic agents. Shock 2002;18: 542-548.

2 Leveckis J, Brown NJ, Reed MWR: The effect of aminolaevulinic acid-induced protoporphyrin IX-mediated photodynamic therapy on the cremaster muscle microcirculation in vivo. Br J Cancer 1995;72:1113-1119.

$\checkmark 3$ Miller FN, Joshua IG, Anderson GL: Quantization of vasodilator-induced macromolecular leakage by in vivo fluorescent microscopy. Microvasc Res 1982;24:56-67.

4 Bingaman S, Huxley VH, Rumbaut RE: Fluorescent dyes modify properties of proteins used in microvascular research. Microcirculation 2003; 10:221-231.

5 Pass HI: Photodynamic therapy in oncology: mechanisms and clinical use. J Natl Cancer Inst 1993;85:443-456.

6 6 Reed MWR, Miller FN: Importance of light dose in fluorescent microscopy. Microvasc Res 1988;36:104-107.
7 Gawlowski DM, Harding NR, Granger HJ Leukocyte phagocytosis and alterations in microvascular integrity elicited by FITCdextran 150 and epi-illumination in the microcirculation of the hamster cheek pouch Microvasc Res 1989;37:1-15.

8 Gawlowski DM, Benoit JN, Granger HJ: Microvascular pressure and albumin extravasation after leukocyte activation in hamster cheek pouch. Am J Physiol 1993;264:H541H546.

-9 Miller FN, Sims DE, Schuschke D, Abney DL: Differentiation of light-dye effects in the microcirculation. Microvasc Res 1992;44: 166-184.

10 Vink H, Duling BR: Identification of distinct luminal domains for macromolecules, erythrocytes, and leukocytes within mammalian capillaries. Circ Res 1996;79:581589.

11 Zhang JL, Yokoyama S, Ohhashi T: Inhibitory effects of fluorescein isothiocyanate photoactivation on lymphatic pump activity. Microvasc Res 1997;54:99-107.
12 Huxley VH, Curry FE, Adanson RH: Quantitative fluorescence microscopy on single capillaries: alpha-lactalbumin transport. Am J Physiol 1987;252:H188-H197.

13 Lichtenbeld HC, Yuan F, Michel CC, Jain RK: Perfusion of single tumor microvessels: application to vascular permeability measurement. Microcirculation 1996;3:349-357.

14 Haugland RP: Handbook of Fluorescent Probes and Research Chemicals. Eugene, Molecular Probes, 1996.

15 Kubes P, Payne D, Ostrovsky L: Preconditioning and adenosine in I/R-induced leukocyte endothelial cell interactions. Am J Physiol 1998;274:H1230-H1238.

16 Kurose I, Kubes P, Wolf R, Anderson DC, Paulson J, Miyasaka M, Granger DN: Inhibition of nitric oxide production: mechanisms of vascular albumin leakage. Circ Res 1993; 73:164-171.

17 Mayhan WG: Role of nitric oxide in modulating permeability of hamster check pouch in response to adenosine $5^{\prime}$-diphosphate and bradykinin. Inflammation 1992;16:295-305. 
18 Huxley VH: What do measures of flux tell us about vascular wall biology? Microcirculation 1998;5:109-116.

-19 Rumbaut RE, Harris NR, Sial AJ, Huxley $\mathrm{VH}$, Granger DN: Leakage response to LNAME differ with the fluorescent dye used to label albumin. Am J Physiol 1999; 276:H333-H339.

-20 Rumbaut RE, Sial AJ: Differential phototoxicity of fluorescent dye-labeled albumin conjugates. Microcirculation 1999;6:205-213.

-21 Yuan F, Leuing M, Berk DA, Jain RK: Microvascular permeability of albumin, vascular surface area, and vascular volume measured in human adenocarcinoma LS174T using dorsal chamber in SCID mice. Microvasc Res 1993;45:269-289.
22 Martin JP, Logsdon N: Oxygen radicals mediate cell inactivation by acridine dyes, fluorescein, and lucifer yellow $\mathrm{CH}$. Photochem Photobiol 1987;46:45-53.

23 McDonagh PF, Williams SK: The preparation and use of fluorescent-protein conjugates for microvascular research. Microvasc Res 1984;27:14-27.

24 Valenzeno DP: Photomodification of biological membranes with emphasis on singlet oxygen mechanisms. Photochem Photobiol 1987;46:147-160.

25 Brookes ZL, Kaufman S: Effects of atrial natriuretic peptide (ANP) on the extra-splenic microvasculature and lymphatics in the rat in vivo. J Physiol 2005;565:269-277.
26 Brown NJ, Pollock KJ, Bayjoo P, Reed MW: The effect of cryotherapy on the cremaster muscle microcirculation in vivo. Br J Cancer 1994;69:706-710.

27 Hutchins PM, Marshburn TH, Maultsby SJ, Lynch CD, Smith TL, Dusseau JW: Longterm microvascular response to hydralazine in spontaneously hypertensive rats. Hypertension 1988;12:74-79.

28 Smith TL, Osborne SW, Hutchins PM: Longterm micro- and macrocirculatory measurements in conscious rats. Microvasc Res 1985; 29:360-370.

29 Boric MP, Roblero JS, Duran WN: Quantitation of bradykinin-induced microvascular leakage of FITC-dextran in rat cremaster muscle. Microvasc Res 1987;33:397-412. 